\title{
Auditory Brainstem Responses \& Nerve Conduction Velocity in Essential Hypertension
}

\author{
Shilpa Khullar*, Navin Gupta and Rashmi Babbar
}

Department of Physiology, Maulana Azad Medical College, Bahadur Shah Zafar Marg, New Delhi-110002, India

\begin{abstract}
Auditory Brainstem Responses (ABRs) is an electrophysiologic technique that represents the synchronized activity of the brainstem and the auditory nerve. Nerve conduction velocity (NCV) is part of electrodiagnostic procedures that help in evaluating the type and degree of abnormalities of the peripheral nerves. The aim of our study was to assess whether central or peripheral nerves are involved in hypertensive patients. 20 patients of primary hypertension were selected between the ages of 40-60 years of either sex along with 20 age- and sex-matched normotensives. ABRs along with $\mathrm{NCV}$, both sensory and motor components of the median nerve were performed using standard techniques. Auditory threshold increased significantly in the hypertensive group compared with controls $(p<0.05)$. There was a significant prolongation of absolute peak latencies of waves I, II and V and interpeak latency III-V. However, no significant difference was observed in the NCV between the hypertensives and controls. We conclude that there was a significant correlation of rise in systolic and diastolic blood pressure with absolute peak latencies of ABRs in hypertensive patients. However, no significant difference in nerve conduction velocity was seen.
\end{abstract}

Keywords: Hypertension, essential hypertension, stimulus related potential, nerve conduction velocity, neuropathy, auditory brainstem response, evoked potential.

\section{INTRODUCTION}

Central nervous system dysfunctions are common in patients of essential hypertension [1]. Arterial and arteriolar spasm in cerebral blood vessels may be responsible [2]. Micro-infarctions are known to be responsible for dysfunction in heart, kidney, brain and other tissues in patients of hypertension. Moreover hypertension appears to accelerate the course of atherosclerosis and increase the incidence of cerebral as well as myocardial infarction [1].

A variety of clinical features of sensory and motor deficit along with the symptoms of dizziness, vertigo, tinnitus and occipital headache in patients of essential hypertension suggests the presence of micro-vascular insufficiency in cerebral tissue [1]. Such type of micro-vascular damage may alter brain stem auditory evoked potentials (BAEPs) which in turn may provide the early evidence for the presence of central nervous system dysfunction in patients of primary hypertension [1]. Therefore, our study was carried out to evaluate the effect of essential hypertension on BAEPs.

Sensory motor deficit in patients with essential hypertension may also be because of micro-vascular damage in peripheral nervous system [1]. Presence of peripheral neuropathy in diabetes mellitus is well documented [1]. Hypertension is one of the factors associated with neuropathy [3] and micro-vascular disease [4] in patients of diabetes mellitus. Although primary etiology of neuropathy is diabetes mellitus

*Address correspondence to this author at the Department of Physiology, Maulana Azad Medical College, Bahadur Shah Zafar Marg, New Delhi110002, India; Tel: 091-11-9968051096;

E-mail: drshilpakhullar@gmail.com in such patients, hypertension has also been identified as a risk factor [4]. Frequent co-existence of hypertension and neuropathy in patients of diabetes mellitus suggests a microvascular basis. Improvement of nerve conduction velocity in diabetics by the use of angiotensin converting enzyme (ACE) inhibitors again suggests the role of essential hypertension in diabetic neuropathy $[5,6]$. Moreover, nerve conduction velocity has also been independently related to pulse pressure in diabetes mellitus [7]. Data on nerve conduction velocity in patients of essential hypertension, without diabetes mellitus, is inconsistent. Reduction in motor conduction velocity in essential hypertension patients was reported [8] but subsequent studies failed to demonstrate such differences $[9,10]$. The present study is an attempt to identify the effect of essential hypertension (without associated diabetes mellitus) on motor as well as sensory conduction velocity of median nerve. This work will be further extended by taking subjects with longer duration of essential hypertension.

\section{METHODS}

This study was conducted in the Neurophysiology laboratory, Dept. of Physiology, Maulana Azad Medical College (MAMC), New Delhi, India. The study protocol was approved by the ethical committee of the institute and extended over a period of 1 year between March, 2004 to March, 2005. A written informed consent was obtained from each participant. Our study comprised of 20 patients with primary hypertension between the ages of 40 and 60 years of either sex along with 20 age- and sex-matched normotensive subjects. Subjects were selected from the outpatient department (OPD) of Lok Nayak Hospital, New Delhi and controls were normotensive volunteers. 


\section{Selection of Hypertensive Subjects}

The criterion of considering a patient hypertensive was a BP $>140 / 90 \mathrm{mmHg}$ [11] based on the average of 2 or more readings taken during each of his/ her visits to the OPD. These subjects were not on any antihypertensive medication and they were not acutely ill. The controls were the staff members of the hospital with Systolic Blood Pressure (SBP) $<120 \mathrm{mmHg}$ and Diastolic Blood Pressure (DBP) $<80$ $\mathrm{mmHg}$ [11]. A detailed form that included clinical history was completed for each subject. These subjects were investigated and diagnosed as cases of essential hypertension. Subjects with any associated diseases like diabetes mellitus, ischemic heart disease (IHD), cerebrovascular disease, renal disease and hearing deficit were excluded from the study. Before putting them on any antihypertensives, they were given a detailed systemic and ENT examination and their hearing threshold was determined using pure tone audiometry (PTA). Auditory Brainstem Responses (ABRs) and Nerve Conduction Velocity (NCV), both sensory and motor components of the median nerve were recorded using computerized evoked potential recording system 'EBNeuro' (Florence, Italy).

\section{Selection of Controls}

The controls were the staff members of the hospital with Systolic Blood Pressure (SBP) $<120 \mathrm{mmHg}$ and Diastolic Blood Pressure (DBP) $<80 \mathrm{mmHg}$ [11]. Exclusion criteria used in this group were similar to the criteria used in hypertensive group. Control group was subjected to the same testing as the hypertensive group simultaneously.

\section{Recording of ABRs}

The subjects were lying down at the time of study in a sound proof room at ambient room temperature between 10 a.m to 12 p.m. $\mathrm{Ag} / \mathrm{AgCl}$ disc electrodes were fixed using conducting paste according to '10-20 International system of electrode placement'. Active electrode was placed at ipsilateral ear lobule (Ai), reference electrode was placed at $\mathrm{Cz}$ and Grounding electrode was placed at the forehead (Fz). Electrical impedance was kept below $5 \mathrm{k} \Omega$. Acoustic transients (alternating clicks) were now delivered through earphones. Each brief click stimulus is a square wave pulse of $0.1 \mathrm{msec}$. A click rate of $11 \mathrm{kHz}$ was used. A total of 1500 individual sweeps were recorded using filter band pass of $300-3000 \mathrm{~Hz}$ with artefact rejection level up to 25 microvolts. 2 to 3 repetitions of the recording were done to ensure reproducibility i.e. - latency measured on separate recordings agreed with each other within $0.1 \mathrm{msec}$ or less. The parameters recorded and analysed included:

1. Absolute latency of all the waves from I to $\mathrm{V}$

2. Interpeak latency I-III, I-V and III-V

3. Amplitude ratio of waves $\mathrm{V}$ and $\mathrm{I}$

\section{Recording of Nerve Conduction Velocity}

Nerve conduction studies were performed on 'EB Neuro' (Italy) system using MYTO CE0051 software. Motor as well as sensory conduction velocity of right median nerve was measured. Subjects were allowed to acclimatize in an air conditioned room $\left(25^{\circ} \mathrm{C}\right)$ for $15 \mathrm{~min}$ before the procedure. Recordings were obtained at following instrument settings:

\section{For Motor Studies (Median Nerve)}

Sensitivity: $2-5 \mathrm{mv} / \mathrm{mm}$, Low frequency filter: $2-5 \mathrm{~Hz}$, High frequency filter: $10 \mathrm{KHz}$, Sweep speed: $2-5 \mathrm{~ms} / \mathrm{mm}$.

\section{For Sensory Studies(Median Nerve)}

Sensitivity: 10-20 $\mu \mathrm{v} / \mathrm{mm}$, Low frequency filter: $5-10 \mathrm{~Hz}$, High frequency filter: 2-3 KHz, Sweep speed: 1-2 ms/min.

Stimulation was done using supra-maximal stimulus with a square wave of $0.1 \mathrm{~ms}$ duration. For motor conduction velocity on median nerve the electro-cutaneous stimulus was delivered to the skin over the median nerve at both the proximal wrist crease, between the central palmaris longus tendon and the flexor carpi radialis tendon, as well as at the elbow crease at the medial aspect of cubital fossa. Impedance at both the sites was kept below $8 \mathrm{k} \Omega$. Latent period was measured as time interval between stimulus artefact and onset of electrical response. Nerve conduction velocity was calculated by dividing the latent period by nerve length. Nerve length was measured using a metal tape. Sensory conduction velocity was determined by giving the stimulus at the base of middle finger.

\section{Statistics}

Student's ' $t$ ' test was used to compare the values between the study and control groups using MS Excel. A P $<0.05$ was considered significant.

\section{RESULTS}

\section{Control Group}

There were 20 subjects in this group between 40 and 60 years of age with average being $51.1 \pm 6.9$ years. They had mean weight: $64.0 \pm 5.6 \mathrm{~kg}$, height: $166.3 \pm 5.6 \mathrm{~cm}$, body surface area: $1.73 \pm 0.09 \mathrm{~kg} / \mathrm{m}^{2}$, SBP: $118 \pm 7.5 \mathrm{mmHg}$ and DBP: $78.3 \pm 6.6 \mathrm{mmHg}$.

\section{Hypertensive Group}

There were 20 subjects in this group with their age between 40 to 60 years, average being $50.2 \pm 5.9$ years. They had an average weight: $67.3 \pm 5.8 \mathrm{~kg}$, height: $166.4 \pm 7.0$ $\mathrm{cm}$, body surface area: $1.75 \pm 0.11 \mathrm{~kg} / \mathrm{m}^{2}$, SBP: $146.2 \pm 16.9$ mmHg and DBP: $98.4 \pm 8.1 \mathrm{mmHg}$. On the basis of extent of raised $\mathrm{BP}$, these subjects belonged to stage 1 and stage 2 hypertension as per criteria laid down by the $\mathrm{VII}^{\text {th }}$ report of the Joint National Committee on selection, evaluation and treatment of high blood pressure [11].

The female subjects both in the control and study groups, had slightly lower values of ABRs as compared with males but as these were not significant, composite data are given in Table 1. Since values of ABRs of left and right ear did not vary significantly, an average of the 2 ears was calculated and their absolute latencies, interpeak latencies (IPLs) and amplitude ratio V/I are given in Table $\mathbf{1}$. On comparing the ABRs between the study and control groups, there was statistically significant prolongation of latency of waves I, II and $\mathrm{V}$, along with interpeak latency III-V in the hypertensives compared to control group $(\mathrm{P}<0.05)$ (Table $\mathbf{1})$. 
Table 1. Absolute Latencies, Inter-Peak Latencies, Amplitude Ratio and Nerve Conduction Velocities in Hypertensives as Compared with Normotensives. Data is Presented as mean \pm SD. * Indicates 'P' $<0.05$

\begin{tabular}{|c|c|c|}
\hline & Normotensives & Hypertensives \\
\hline I & $1.7 \pm 0.14$ & $1.85 \pm 0.12 *$ \\
\hline II & $2.72 \pm 0.16$ & $2.91 \pm 0.18^{*}$ \\
\hline III & $3.72 \pm 0.16$ & $3.79 \pm 0.11$ \\
\hline $\mathrm{V}$ & $5.61 \pm 0.15$ & $5.78 \pm 0.14$ \\
\hline \multicolumn{3}{|l|}{ Inter-peak latency (ms) } \\
\hline I-III & $1.99 \pm 0.16$ & $2.01 \pm 0.18$ \\
\hline Motor conduction velocity $(\mathrm{m} / \mathrm{s})$ & $59.63 \pm 3.27$ & $58.32 \pm 3.48$ \\
\hline Sensory conduction velocity $(\mathrm{m} / \mathrm{s})$ & $61.13 \pm 3.84$ & $60.08 \pm 3.92$ \\
\hline
\end{tabular}

Motor as well as sensory conduction velocities were not significantly different in hypertensives as compared with normotensives $(\mathrm{P}>0.05)$ (Table $\mathbf{1})$.

\section{DISCUSSION}

The aim of our study was to evaluate the effect of hypertension on ABRs and conduction velocity of the median nerve, both sensory and motor components. 20 patients with primary hypertension were selected between the ages of 40 and 60 years along with 20 age- and sex-matched normotensives. In our study the auditory threshold increased significantly among the study group. The absolute peak latency of waves I, II and V was significantly higher in hypertensives compared to controls $(\mathrm{P}<0.05)$. In addition IPL III-V was significantly prolonged in the study group compared with controls, but there was no difference in the conduction velocity between the 2 groups as reported in similar studies [9, 10].

Our study though preliminary shows sensory dysfunction of the auditory pathway at the brainstem level in hypertensives. Our findings are similar to those of a study that reported prolongation of latency of all waves of ABR along with IPL III-V [12]. In another study, 55 essential hypertensive patients and 55 normal elderly subjects between the ages of 55-99 years were selected. ABRs were measured along with serum cholesterol and triglyceride levels. The results of the ABR demonstrated that the latencies of wave V, IPL I-V and IPL III-V were prolonged compared with normal elderly subjects. The patient's duration of illness and the complications of hypertension had an influence on hearing disorders in relation to the ageing process. Hence they concluded that the hearing disorders in the elderly people are a result of long duration of illness and the complications of hypertension [13].

Karamitsos et al. studied ABRs in 30 patients of ischaemic heart disease and in an equal number of healthy agematched control subjects. The parameters measured were absolute latency of waves I through $\mathrm{V}$, the interpeak latencies I-III, III-V, and I-V, and the peak amplitudes of wave I, III, and V. The measured absolute latencies and interpeak latencies were found to be significantly increased, and the peak amplitudes were found to be diminished in the study group. Hence BAEPs may become part of the noninvasive assessment IHD and essential hypertension patients [14].

Auditory brainstem-evoked responses were conducted on 28 patients with otologic symptoms (pulsatile tinnitus, hearing loss, aural fullness) secondary to benign intracranial hypertension syndrome. Abnormalities consisting mainly of prolonged interpeak latencies were detected in one third of these patients. It is speculated that the pathophysiologic mechanisms responsible for these auditory brainstem-evoked abnormalities are stretching-compression of the cochlear nerve in the brainstem, caused by the intracranial hypertension and/or primary edema. Normalization or improvement was noticed in the majority of the patients after management of intracranial hypertension. Since the number of patients in this study is small, the diagnostic and prognostic value of this test needs further evaluation [15]. Significant prolongation of waves IV, V and VII of ABRs were also seen in rabbits with intra-cranial hypertension compared with controls [16].

The findings of the present study further support the notion that a rise in blood pressure affects the sensory conduction in the auditory pathway in the brainstem. Another study [17] showed that raised blood pressure in pre-eclamptic 
women may affect the vascular responses of the blood vessels in brain and cause ischaemic delay in P1 latency of visual evoked potentials. This may also explain the delay in ABR latencies in hypertensive cases. Correlation between metabolic disorders involving myelination and abnormality in ABRs has not been studied. Further work is required to find the metabolic basis of our findings.

The present study examined the motor as well as sensory conduction velocity of median nerve in essential hypertensive patients. No significant difference was found in nerve conduction velocity (both sensory and motor) of hypertensives compared with normotensives.

Evidence on nerve conduction studies in hypertension is not consistent. Motor conduction velocity in the upper limb was found to be lower in hypertensives as compared with normotensives [8]. Improvement in nerve conduction velocity, temperature discrimination threshold and vibration perception threshold after treatment with a blood pressure lowering agent, lisinopril, in patients of diabetes mellitus [6] again suggests the role of hypertension in the development of neuropathy in these patients. Improved nerve conduction velocity by treatment with an ACE inhibitor (trandolapril) in diabetes mellitus patients was also reported [5], although they failed to find any improvement in vibration perception threshold, autonomic function and neuropathy symptoms even after 12 months of therapy. Increase in nerve conduction velocity by lisinopril has also been demonstrated in streptozotocin-diabetic rats [18, 19]. Further, pulse pressure has been shown to be independently associated with peripheral neuropathy in patients of diabetes mellitus [7].

Animal studies are also consistent with development of neuropathy with hypertension. Reduced vascular supply to peripheral nerves because of luminal narrowing was demonstrated in spontaneously hypertensive rats [20] which were reverted with anti hypertensive treatment [21]. Apart from a reduction in nerve conduction velocity, decreased percentage of class I fibers and increased percentage of class III and IV fibers was also found in sciatic nerve of spontaneously hypertensive rats [22]. These changes were reversed by anti hypertensive treatment with hydralazine [22].

Contrary to these results we did not find any difference in motor as well as sensory conduction velocity of median nerve in hypertensives as compared with normotensives. After demonstration of reduced nerve conduction velocity by Viscoper [8] and co-workers in 1971, subsequent studies failed to demonstrate the same $[9,10]$. Motor and sensory conduction velocities of median nerve in essential hypertensives who were not on any medication, were also similar as compared with normotensives [23]. They performed the study in un-medicated patients to rule out any effect of drugs. However, they detected reduction in sensory action potential amplitude and cutaneous sensitivity in hypertensives. They proposed that hypertension may be producing axonal degeneration but not affecting myelination, thereby preserving nerve conduction velocity. Further, beneficial effect of ACE inhibitors on nerve conduction velocity has only been demonstrated in diabetic neuropathy patients. Any such effect in the absence of diabetes mellitus is not known [24].
We conclude that essential hypertension per se influences the ABRs but does not produce any effect on nerve conduction velocity. Hypertension might be affecting central conduction but peripheral myelination is preserved. Further studies are required in patients with longer duration of hypertension.

\section{REFERENCES}

[1] Williams GH. In: Isselbacher KJ, Braunwald E, Wilson JD, Martin JB, Fauci AS, Kasper DL Eds, Harrisons principles of internal medicine $13^{\text {th }}$ ed. New York, McGraw-Hill Inc 1994; 1116-31.

[2] Frederic MW. In: Conn HL, Horwitz O Eds, Cardiac and vascular diseases. Philadelphia, Lea and Febiger 1971; 1473-99.

[3] Tesfaye S, Chaturvedi N, Eaton SEM, et al. Vascular risk factors and diabetic neuropathy. N Engl J Med 2005; 352: 341-50.

[4] Forrest KYZ, Maser RE, Pambianco G, et al. Hypertension as a risk factor for diabetic neuropathy - A prospective study. Diabetes 1997; 46: 665-70.

[5] Malik RA, Williamson S, Abbott C, et al. Effect of angiotensin converting - enzyme (ACE) inhibitor trandolapril on human diabetic neuropathy: randomized double blind controlled trial. Lancet 1998; 352: 1978-81.

[6] Reja A, Tesfaye S, Harris ND, et al. Is ACE inhibition with lisinopril helpful in diabetic neuropathy? Diabet Med 1995; 12: 307-9.

[7] Jarmuzewska EA, Mangoni AA. Pulse pressure is independently associated with sensorimotor peripheral neuropathy in patients with type 2 diabetes. J Intern Med 2005; 258: 38-44.

[8] Viskoper RJ, Chaco J, Aviram A. Nerve conduction velocity in assessment of hypertension. Arch Intern Med 1971; 128: 574-5.

[9] Bridgman JF, Bidgood L, Hoole R. Nerve-conduction velocity and hypertension. Br Med J 1973; 3: 500-1.

[10] Halar EM, Stewart DT, Venkatesh B, et al. Nerve conduction velocity in hypertensive patients. Arch Intern Med 1978; 138: 121-3.

[11] The seventh report of the Joint National Committee on detection, evaluation and treatment of high blood pressure (JNC-VII). Hypertension 2003; 42: 1206.

[12] Tandon OP, Ram D, Awasthi R. Brainstem auditory evoked responses in primary hypertension. Indian J Med Res 1996; 104: 311 5.

[13] Chen YL, Ding YP. Relationship between hypertension and hearing disorders in the elderly. East Afr Med J 1999; 76: 344-7.

[14] Karamitsos DG, Kounis NG, Zavras GM, et al. Brainstem auditory evoked potentials in patients with ischemic heart disease. Laryngoscope 1996; 106: 54-7.

[15] Sismanis A, Callari RH, Slomka WS, et al. Auditory-evoked responses in benign intracranial hypertension syndrome. Laryngoscope 1990; 100: 1152-5.

[16] Wang J, Liu YS, Liu SM. Changes in somatosensory evoked potentials and brainstem auditory evoked potentials during acute intracranial hypertension in rabbits. Hunan Yi Ke Da Xue Xue Bao 2001; 26: 197-9.

[17] Marsh MS, Smith S. The visual evoked potential in the assessment of central nervous effects of pre-eclampsia - a pilot study. Br J Obstet Gynaecol 1994; 101: 343-6.

[18] Cameron NE, Cotter MA, Robertson S. Rapid reversal of a motor nerve conduction deficit in streptozotocin-diabetic rats by the angiotensin converting enzyme inhibitor lisinopril. Acta Diabetol 1993; 30: 46-8.

[19] Aggarwal M, Singh J, Sood S, et al. Effects of lisinipril on streptozotocin-induced diabetic neuropathy in rats. Methods Find Exp Clin Pharmacol 2001; 23: 131-4.

[20] Sabbatini M, Vega JA, Amenta F. Peripheral nerve vascular changes in spontaneously hypertensive rats. Neurosci lett 1996; 217: 85-8.

[21] Sabbatini M, Bellagamba G, Vega JA, et al. Effect of antihypertensive treatment on peripheral nerve vasculature on spontaneously hypertensive rats. Clin Exp Hypertens 2001; 23: 157-66.

[22] Tomassoni D, Traini E, Vitaioli L, et al. Morphological and conduction changes in the sciatic nerve of spontaneously hypertensive rats. Neurosci lett 2004; 362: 131-5. 
[23] Morandini G, Ronchi O, Rossi L, et al. Motor nerve conduction velocity in arterial hypertension. Minerva Med 1976; 67: 3317-23.
[24] Edwards L, Ring C, McIntyre D, et al. Cutaneous sensibility and peripheral nerve function in patients with unmedicated essential hypertension. Psychophysiology 2008; 45: 141-7.

Received: June 05, 2008

Revised: June 25, 2008

Accepted: September 19, 2008

(c) Khullar et al.; Licensee Bentham Open.

This is an open access article licensed under the terms of the Creative Commons Attribution Non-Commercial License (http://creativecommons.org/ licenses/by-nc/3.0/) which permits unrestricted, non-commercial use, distribution and reproduction in any medium, provided the work is properly cited. 